\title{
Oral analgesics after third molar surgery - a systematic review of relative efficacy
}

\author{
Relative efficacy of oral analgesics after third molar extraction
}

\section{J. Barden, J. E. Edwards, H. J. McQuay, P. J. Wiffen and R. Andrew Moore Br Dent J 2004; 197: 407-411}

\section{Objectives}

To compare the relative efficacy of analgesics after third molar extraction from systematic reviews of randomised, double blind studies.

\section{Data sources}

Dental trials from systematic reviews of randomised, double-blind studies of analgesics in acute pain.

\section{Data selection}

Number of patients with moderate or severe pain achieving at least half pain relief over 4 to 6 hours after a single oral dose of analgesic.

\section{Data extraction}

Independently by two reviewers.

\section{Data synthesis}

Use of dichotomous information from active and placebo treatments, first to calculate the statistical significance using relative risk, and then to evaluate the clinical relevance using number needed to treat (NNT). Non-steroidal anti-inflammatory drugs (NSAIDs) and cyclo-oxygenase-2 (COX-2) inhibitors had the lowest (best) NNTs for the outcome of at least half pain relief over 4-6 hours compared with placebo. With the best performing analgesics, 50-70 patients out of 100 had good pain relief compared with about 10 out of 100 with placebo. Only paracetamol 600/650 $\mathrm{mg}$ plus codeine $60 \mathrm{mg}$ was associated with any significant increase in any patient experiencing an adverse event.

\section{Conclusions}

NSAIDs and COX-2 inhibitors have the lowest (best) NNTs. They may also have fewer adverse effects after third molar surgery, though conclusive evidence is lacking. At least $80 \%$ of analgesic prescribing by UK dentists is in line with the best available evidence on efficacy and safety.

\section{IN BRIEF}

- This paper reviews the available high quality information on analgesics commonly prescribed by dentists, including COX-2 selective inhibitors.

- Problems related to chance effects are avoided by combining multiple trials in a meta-analysis.

- There is good evidence of efficacy for most commonly-prescribed analgesics.

- Standard doses of NSAIDs and COX-2 inhibitors provide the best analgesia and lowest rate of adverse events.

\section{COMMENT}

Analgesic usage in dentistry is mainly restricted to the control of post-operative pain after a dental surgical procedure. Of these latter events, third molar surgery is one of the most widely performed dental surgical operations and also an extensively used model to evaluate analgesic efficacy. The authors of this current paper have completed a systematic review of a large number of randomised, double-blind studies that have evaluated the efficacy of a number of analgesics in the control of post-operative pain after third molar surgery. Because of the nature of the pain and its relative short duration, the review is based upon single dose studies. In addition to evaluating efficacy, the authors also considered unwanted effects arising from particular analgesic usage.

The results of their analysis showed that Ibuprofen, diclofenac and the new COX-2 inhibitors (valdecoxib and rofecoxib) offered distinct advantages over drugs such as aspirin, paracetamol and dihydrocodeine. Furthermore, the superior efficacy of these compounds was also supported by a reduced risk of unwanted effects.

Do these findings come as a surprise? At one level the answer has to be no. The pain model used is ideally for evaluating antiinflammatory actions. Both ibuprofen and the new COX-2 inhibitors possess well established anti-inflammatory activity which is difficult to differentiate from the drug's analgesic properties. The advent of the COX-2 inhibitors has generated renewed interest in the third molar pain model. These drugs are designed to have all the benefits of aspirin and NSAIDs without the unwanted effects. Although fewer trials have been completed with these drugs than with ibuprofen, the early impression is that they are a useful addition to the range of analgesics that dentists can prescribe for the control of post-operative pain.

This systematic review has been a useful exercise and adds, or at least confirms, our views on analgesic prescribing and efficacy. The positive outcomes are that ibuprofen and COX-2 inhibitors are the drugs of choice. The review also confirms that dihydrocodeine and paracetamol/codeine combinations are of little or no value. This begs the question as to why a drug like dihydrocodeine continues to remain on the Dental Practitioner's Formulary.

\section{R. A. Seymour, Dean of Dentistry, School of Dental Sciences,} University of Newcastle upon Tyne doi:10.1038/sj.bdj.4811718 